\title{
Generalized Morrey Spaces for Non-doubling Measures
}

\author{
Yoshihiro Sawano
}

\begin{abstract}
In this paper, we define the generalized Morrey spaces on $\mathbb{R}^{d}$ with the measure $\mu$ non-doubling. After defining the space, we shall investigate the properties of maximal operators, fractional integral operators and the singular integral operators. And we shall allude to the vector-valued extension of these operators.
\end{abstract}

Mathematics Subject Classification (2000). Primary 42B35; Secondary 42B25.

Keywords. Generalized Morrey space, non-doubling (nonhomogeneous).

\section{Introduction}

The aim of this paper is to investigate the properties of the generalized Morrey spaces in $\mathbb{R}^{d}$. The Morrey space was defined by C. Morrey in 1938 [7]. Nowadays the Morrey norm is transformed into the one that is handy to deal with. One of the form of this norm is

$$
\left\|f: \mathcal{M}_{q}^{p}\right\|:=\sup _{x \in \mathbb{R}^{d}, r>0} r^{\frac{n}{p}-\frac{n}{q}}\left(\int_{B(x, r)}|f(y)|^{q} d \mu(y)\right)^{\frac{1}{q}},
$$

where $B(x, r)$ is a ball centered at $x$ of radius $r>0$. One can regard $p$ as a global regularity index of the functions. That is, the parameter $p$ seems to reflect the speed of decay at infinity. Meanwhile $q$ corresponds to the local regularity. The generalized Morrey norm, investigated in $[5,6,9]$, generalized the global regularity parameter $p$ : More precisely, let $\phi:(0, \infty) \rightarrow(0, \infty)$ be an increasing function and

In writing this paper, the author is supported financially by Research Fellowships of the Japan Society for the Promotion of Science for Young Scientists. 
$1 \leq p<\infty$. Then we define the generalized Morrey norm of the function $f$ by

$$
\left\|f: \mathcal{L}^{p, \phi}\right\|:=\sup _{x \in \mathbb{R}^{d}, r>0}\left(\frac{1}{\phi(|B(x, r)|)} \int_{B(x, r)}|f(y)|^{p} d \mu(y)\right)^{\frac{1}{p}},
$$

where $|E|$ denotes its measure of a measurable set $E$.

The aim of this paper is to investigate the generalized Morrey space with the underlying measure non-doubling. A measure $\mu$ is said to be a doubling, if there exists $c>0$ so that for every $x \in \mathbb{R}^{d}$ and $\ell>0$

$$
\mu(B(x, 2 \ell)) \leq c \mu(B(x, \ell)) .
$$

The analysis had been difficult, if (2) fails. However, due to the pioneering works by Nazarov, Treil, Volberg and Tolsa $[10,11,17,18]$ people gained a new insight of this field. Their modification theory was a key to overcome the difficulty in dealing with non-doubling measures.

In what follows "by cube" we mean a compact set of the form

$$
Q:=\left[a_{1}-l, a_{1}+l\right] \times \cdots \times\left[a_{d}-l, a_{d}+l\right]
$$

and we denote by $\mathcal{Q}(\mu)$ the set of all doubling cubes with positive $\mu$-measure. In [15], we define the Morrey norm of a function $f$ as follows:

$$
\left\|f: \mathcal{M}_{q}^{p}(k, \mu)\right\|:=\sup _{Q \in \mathcal{Q}(\mu)} \mu(k Q)^{\frac{1}{p}-\frac{1}{q}}\left(\int_{Q}|f|^{q} d \mu\right)^{\frac{1}{q}} .
$$

Let $1 \leq q \leq p<\infty$. In [15, Proposition 1.1] we have shown that the parameter $k>1$ does not affect the set of $\mathcal{M}_{q}^{p}(k, \mu)$. More precisely, let $k_{1}>k_{2}>1$. Then there is a constant $C_{d}$ depending only on $d$ and $k_{1}, k_{2}$ so that, for every $\mu$-measurable function $f$,

$$
\left\|f: \mathcal{M}_{q}^{p}\left(k_{1}, \mu\right)\right\| \leq\left\|f: \mathcal{M}_{q}^{p}\left(k_{2}, \mu\right)\right\| \leq C_{d}\left(\frac{k_{2}-1}{k_{1}-1}\right)^{d}\left\|f: \mathcal{M}_{q}^{p}\left(k_{1}, \mu\right)\right\| .
$$

Thus, $\mathcal{M}_{q}^{p}\left(k_{1}, \mu\right)$ and $\mathcal{M}_{q}^{p}\left(k_{2}, \mu\right)$ coincide as a set and their norms are mutually equivalent.

Motivated by this fact, we define the generalized Morrey spaces as follows:

Definition 1.1. Let $1 \leq p<\infty$. Suppose that $\phi:(0, \infty) \rightarrow(0, \infty)$ is an increasing function. Then define

$$
\left\|f: \mathcal{L}^{p, \phi}(k, \mu)\right\|:=\sup _{Q \in \mathcal{Q}(\mu)}\left(\frac{1}{\phi(\mu(k Q))} \int_{Q}|f(x)|^{p} d \mu(x)\right)^{\frac{1}{p}} .
$$

$\mathcal{L}^{p, \phi}$ is a set of $L_{l o c}^{p}(\mu)$ functions $f$ for which the norm $\left\|f: \mathcal{L}^{p, \phi}(\mu)\right\|$ is finite.

As we did in [15, Proposition 1.1], by bisecting cubes, we can prove the following proposition. 
Proposition 1.2. Let $k_{1}>k_{2}>1$ and $1 \leq p<\infty$. Suppose that $\phi:(0, \infty) \rightarrow$ $(0, \infty)$ is an increasing function. Then there exists a constant $C_{d}$ depending only on $k_{1}, k_{2}, d$ so that

$$
\left\|f: \mathcal{L}^{p, \phi}\left(k_{1}, \mu\right)\right\| \leq\left\|f: \mathcal{L}^{p, \phi}\left(k_{2}, \mu\right)\right\| \leq C_{d}\left(\frac{k_{2}-1}{k_{1}-1}\right)^{d}\left\|f: \mathcal{L}^{p, \phi}\left(k_{1}, \mu\right)\right\| .
$$

Thus, $\mathcal{L}^{p, \phi}\left(k_{1}, \mu\right)$ and $\mathcal{L}^{p, \phi}\left(k_{2}, \mu\right)$ coincide as a set and their norms are mutually equivalent.

Keeping Proposition 1.2 in mind, below we shall denote $\mathcal{L}^{p, \phi}(\mu)=\mathcal{L}^{p, \phi}(2, \mu)$. Nevertheless, it is important to vary the modification parameter $k>1$.

Finally we shall describe the organization of this paper. In Section 2, we shall prove the boundedness of the maximal operator. In Section 3, we take up the fractional integral operators. Until the meddle of Section 3, we do not postulate anything on $\mu$ : $\mu$ is just a Radon measure. We are still able to define so called "Riesz potential" for a Radon measure in general. In $[13,14]$ we have defined a fractional integral operator that plays a role of a majorant operator of the fractional maximal operators. If in addition $\mu$ satisfies the growth condition

$$
\mu(B(x, \ell)) \leq c_{0} \ell^{n}, \quad 0<n \leq d,
$$

then it also majorize $I_{\alpha}$ defined in [2, Chapter 6$]$, where $I_{\alpha}$ is given by

$$
I_{\alpha} f(x)=\int_{\mathbb{R}^{d}} \frac{f(y)}{|x-y|^{n-\alpha}} d \mu(y) .
$$

We also remark that García-Cuerva and Gatto showed that $I_{\alpha}$ raises the regularity of functions [3]. Finally in Section 4, the boundedness of the singular integral operators with $\mu$ satisfying (5) will be established. We also note that the letter $c>0$ will be used for constants that may change from one occurrence to another.

\section{Maximal inequalities}

In this section we investigate the boundedness property of the maximal operator given by

$$
M f(x)=\sup _{x \in Q \in \mathcal{Q}(\mu)} \frac{1}{\mu(2 Q)} \int_{Q}|f(y)| d \mu(y) .
$$

\subsection{Preliminaries}

Before investigating the boundedness of the maximal operator, we shall recall an elementary fact on the assumption of the function $\phi$.

The following lemma ensures that the integrablity of the functions can be boostered automatically.

Lemma 2.1 ([9, Lemma 2]). Suppose that $\psi:(0, \infty) \rightarrow(0, \infty)$ be a function satisfying

$$
\int_{r}^{\infty} \psi(s) \frac{d s}{s} \leq c \psi(r) \quad \text { for all } \quad r>0 .
$$


Then there exists $\varepsilon>0$ so that $\int_{r}^{\infty} \psi(s) s^{\varepsilon} \frac{d s}{s} \leq c \psi(r) r^{\varepsilon}$ for all $r>0$. In particular for every $\eta \leq 1$ there exists $c>0$ so that $\int_{r}^{\infty} \psi(s)^{\eta} \frac{d s}{s} \leq c \psi(r)^{\eta}$ for all $r>0$.

We shall need a covering lemma below for later consideration.

Lemma 2.2 ([13, Lemma 12]). Let $b>a>0$ be fixed positive numbers. Suppose that $\mu$ is a Radon measure and

$$
\mathcal{Q}_{a, b}:=\left\{Q \in \mathcal{Q}(\mu): a \leq \mu\left(\kappa^{2} Q\right) \leq b\right\} \neq \emptyset .
$$

Then there exists $N\left(=N_{\kappa}\right)$ subfamilies $\mathcal{Q}(\mu)_{a, b, 1}, \ldots, \mathcal{Q}(\mu)_{a, b, N}$ such that

$$
\left\{\kappa Q: Q \in \mathcal{Q}(\mu)_{a, b, j}\right\} \quad \text { is disjoint for all } j=1, \ldots, N \text {. }
$$

and for all $Q \in \mathcal{Q}(\mu)_{a, b}$ we can find $Q^{\prime} \in \bigcup_{j=1}^{N} \mathcal{Q}(\mu)_{a, b, j}$ such that $Q \subset \kappa Q^{\prime}$. Here $N_{\kappa}$ does not depend on a nor $b$.

\subsection{Boundedness of the maximal operator $M$}

It is well-known that $M$ is bounded on $L^{p}(\mu), 1<p \leq \infty$. For details we refer to [11]. We present the main theorem in this section.

Theorem 2.3. Suppose that $\phi:(0, \infty) \rightarrow(0, \infty)$ is an increasing function. Assume that the mapping $t \mapsto \frac{\phi(t)}{t}$ is almost decreasing: There exists a constant $c>0$ such that

$$
\frac{\phi(t)}{t} \leq c \frac{\phi(s)}{s}
$$

for $s \geq t$. Then there exists a constant $c>0$ so that, for every $f \in \mathcal{L}^{p, \phi}(\mu)$,

$$
\left\|M f: \mathcal{L}^{p, \phi}(\mu)\right\| \leq c\left\|f: \mathcal{L}^{p, \phi}(\mu)\right\| .
$$

Proof. Let $Q \in \mathcal{Q}(\mu)$ be a fixed cube. Then it suffices to establish

$$
\left(\frac{1}{\phi(\mu(10 Q))} \int_{Q} M f(x)^{p} d \mu(x)\right)^{\frac{1}{p}} \leq c\left\|f: \mathcal{L}^{p, \phi}(\mu)\right\| .
$$

We decompose $f$ according to $8 Q$. That is, we split $f=f_{1}+f_{2}$ with $f_{1}=\chi_{8 Q} f$. The estimate of $f_{1}$ is simple. Since $M$ is bounded on $L^{p}(\mu)$, we have

$$
\begin{aligned}
& \left(\frac{1}{\phi(\mu(10 Q))} \int_{Q} M f_{1}(x)^{p} d \mu(x)\right)^{\frac{1}{p}} \\
& \quad \leq c\left(\frac{1}{\phi(\mu(10 Q))} \int_{8 Q}|f(x)|^{p} d \mu(x)\right)^{\frac{1}{p}} \leq c\left\|f: \mathcal{L}^{p, \phi}(\mu)\right\| .
\end{aligned}
$$

Thus, the estimate for $f_{1}$ is valid. Next, we turn our attention to $f_{2}$. A geometric observation shows, for every $x \in Q$,

$$
M f_{2}(x)=\sup _{x \in R \in \mathcal{Q}} \frac{1}{\mu(2 R)} \int_{R}\left|f_{2}(y)\right| d \mu(y) \leq \sup _{x \in R \in \mathcal{Q} Q \subset R} \frac{1}{\mu\left(\frac{4}{3} R\right)} \int_{R}|f(y)| d \mu(y) .
$$


Therefore, we obtain

$$
\begin{aligned}
\left(\frac{1}{\phi(\mu(10 Q))}\right. & \left.\int_{Q} M f_{2}(x)^{p} d \mu(x)\right)^{\frac{1}{p}} \\
& \leq\left(\frac{\mu(Q)}{\phi(\mu(10 Q))}\right)^{\frac{1}{p}} \sup _{\substack{x \in R \in \mathcal{Q} \\
Q \subset R}} \frac{1}{\mu\left(\frac{4}{3} R\right)} \int_{R}|f(y)| d \mu(y) \\
& \leq\left(\frac{\mu(Q)}{\phi(\mu(Q))}\right)^{\frac{1}{p}} \sup _{\substack{x \in R \in \mathcal{Q} \\
Q \subset R}}\left(\frac{\phi\left(\mu\left(\frac{4}{3} R\right)\right)}{\mu\left(\frac{4}{3} R\right)}\right)^{\frac{1}{p}}\left(\frac{1}{\phi\left(\mu\left(\frac{4}{3} R\right)\right)} \int_{R}|f(y)|^{p} d \mu(y)\right)^{\frac{1}{p}} \\
& \leq c\left(\frac{1}{\phi\left(\mu\left(\frac{4}{3} R\right)\right)} \int_{R}|f(y)|^{p} d \mu(y)\right)^{\frac{1}{p}} \\
& \leq c\left\|f: \mathcal{L}^{p, \phi}(\mu)\right\| .
\end{aligned}
$$

Thus, the above chain of inequalities gives us

$$
\left(\frac{1}{\phi(\mu(10 Q))} \int_{Q} M f_{2}(x)^{p} d \mu(x)\right)^{\frac{1}{p}} \leq\left\|f: \mathcal{L}^{p, \phi}(\mu)\right\|,
$$

which is the desired estimate for $f_{2}$.

Remark 2.4. A suitable modification of the above proof allows us to prove the results for the parametrized maximal operator $M_{k}, k>1$, which is given by

$$
M_{k} f(x)=\sup _{x \in Q \in \mathcal{Q}(\mu)} \frac{1}{\mu(k Q)} \int_{Q}|f(y)| d \mu(y) .
$$

\subsection{Vector-valued extension}

Theorem 2.5. In addition to the assumption of Theorem 2.3 suppose that $\phi$ satisfies

$$
\int_{r}^{\infty} \frac{\phi(t)}{t} \frac{d t}{t} \leq c \frac{\phi(r)}{r}
$$

Then, for $1<r \leq \infty$, we have

$$
\left\|\left(\sum_{j \in \mathbb{N}} M f_{j}^{r}\right)^{\frac{1}{r}}: \mathcal{L}^{p, \phi}(\mu)\right\| \leq c\left\|\left(\sum_{j \in \mathbb{N}}\left|f_{j}\right|^{r}\right)^{\frac{1}{r}}: \mathcal{L}^{p, \phi}(\mu)\right\| .
$$

Proof. Fix a cube $Q$ as before, we shall prove

$$
\left(\frac{1}{\phi(\mu(10 Q))} \int_{Q}\left(\sum_{j \in \mathbb{N}} M f_{j}(x)^{r}\right)^{\frac{q}{r}} d \mu(x)\right)^{\frac{1}{q}} \leq c\left\|\left(\sum_{j \in \mathbb{N}}\left|f_{j}\right|^{r}\right)^{\frac{1}{r}}: \mathcal{L}^{p, \phi}(\mu)\right\| .
$$


We decompose $f_{j} j$ according to $8 Q$. We split $f_{j}$ by $f=f_{j, 1}+f_{j, 2}$ with $f_{j, 1}=$ $\chi_{8 Q} f \cdot f_{j}$ and $f_{j, 2}=\chi_{\mathbb{R}^{d} \backslash 8 Q} \cdot f_{j}$. Then for the estimate of the $f_{j, 1}$ we use

$$
\left\|\left(\sum_{j \in \mathbb{N}} M f_{j}^{r}\right)^{\frac{1}{r}}: L^{p}(\mu)\right\| \leq c\left\|\left(\sum_{j \in \mathbb{N}}\left|f_{j}\right|^{r}\right)^{\frac{1}{r}}: L^{p}(\mu)\right\|,
$$

which is established in [12, Theorem 1.7]. The estimate for the $f_{j, 1}$ is now valid by virtue of this inequality.

In order to deal with the $f_{j, 2}$, we shall make use of the following pointwise estimate, which is given by a simple geometric observation:

$$
M f_{j, 2}(x) \leq \sup _{\substack{R \in \mathcal{Q}(\mu) \\ 2 Q \subset R}}\left(\frac{1}{\mu\left(\frac{3}{2} R\right)} \int_{R}\left|f_{j}(y)\right| d \mu(y)\right) \quad \text { for all } \quad x \in Q .
$$

Now for $j \in \mathbb{N}$, we fix a cube $R_{j} \in \mathcal{Q}(\mu)$ so that it satisfies

$$
M f_{j, 2}(x) \leq \frac{2}{\mu\left(\frac{3}{2} R_{j}\right)} \int_{R_{j}}\left|f_{j}(y)\right| d \mu(y) \quad \text { for all } \quad x \in Q .
$$

Let $l \in \mathbb{N}$ be fixed and define

$$
J_{l}:=\left\{j \in \mathbb{N}: 2^{l-1} \mu(2 Q) \leq \mu\left(\frac{3}{2} R_{j}\right)<2^{l} \mu(2 Q)\right\} .
$$

Then by the Minkowski inequality, we have

$$
\begin{aligned}
\left(\sum_{j \in \mathbb{N}} M f_{j, 2}(x)^{r}\right)^{\frac{1}{r}} & \leq 2\left(\sum_{j \in \mathbb{N}}\left(\frac{1}{\mu\left(\frac{3}{2} R_{j}\right)} \int_{R_{j}}\left|f_{j}(y)\right| d \mu(y)\right)^{r}\right)^{\frac{1}{r}} \\
& \leq 2 \sum_{l \in \mathbb{N}}\left(\sum_{j \in J_{l}}\left(\frac{1}{\mu\left(\frac{3}{2} R_{j}\right)} \int_{R_{j}}\left|f_{j}(y)\right| d \mu(y)\right)^{r}\right)^{\frac{1}{r}} \\
& \leq 4 \sum_{l \in \mathbb{N}}\left(\sum_{j \in J_{l}}\left(\frac{1}{2^{l} \mu(2 Q)} \int_{R_{j}}\left|f_{j}(y)\right| d \mu(y)\right)^{r}\right)^{\frac{1}{r}} .
\end{aligned}
$$

If we invoke Lemma 2.2, for each $l$ we can find a subset $J_{l}^{*} \subset J_{l}$ so that

$$
\bigcup_{j \in J_{l}} R_{j} \subset \bigcup_{k \in J_{l}^{*}} \frac{3}{2} R_{k}, \quad \sharp J_{l}^{*} \leq N,
$$


where $N$ depends only on the dimension $d$. Therefore, via this covering, we have

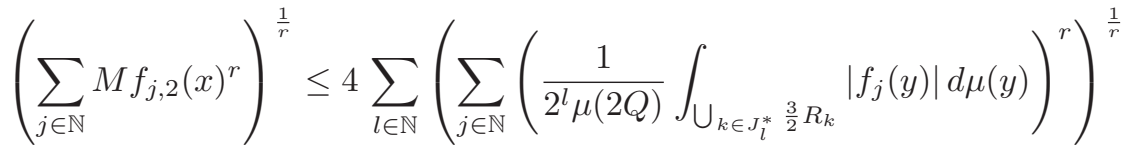

$$
\begin{aligned}
& \leq 4 \sum_{l \in \mathbb{N}} \frac{1}{2^{l} \mu(2 Q)} \int_{\bigcup_{k \in J_{l}^{*}} \frac{3}{2} R_{k}}\left(\sum_{j \in \mathbb{N}}\left|f_{j}(y)\right|^{r}\right)^{\frac{1}{r}} d \mu(y) \\
& \leq 4 \sum_{l \in \mathbb{N}} \sum_{k \in J_{l}^{*}} \frac{\mu\left(\frac{3}{2} R_{k}\right)^{1-\frac{1}{p}}}{2^{l} \mu(2 Q)}\left(\int_{\frac{3}{2} R_{k}}\left(\sum_{j \in \mathbb{N}}\left|f_{j}(y)\right|^{r}\right)^{\frac{p}{r}} d \mu(y)\right)^{\frac{1}{p}} \\
& \leq c \sum_{l \in \mathbb{N}}\left(\frac{\phi\left(2^{l} \mu(2 Q)\right)}{\left.2^{l} \mu(2 Q)\right)}\right)^{\frac{1}{p}}\left\|\left(\sum_{j \in \mathbb{N}}\left|f_{j}\right|^{r}\right)^{\frac{1}{r}}: \mathcal{L}^{p, \phi}(\mu)\right\| .
\end{aligned}
$$

By the assumption (8) and Lemma 2.1, we have

$$
\sum_{l \in \mathbb{N}}\left(\frac{\phi\left(2^{l} \mu(2 Q)\right)}{\left.2^{l} \mu(2 Q)\right)}\right)^{\frac{1}{p}} \leq c\left(\frac{\phi(\mu(2 Q))}{\mu(2 Q))}\right)^{\frac{1}{p}} .
$$

Substituting this and integrating (9) over $Q$, we obtain

$$
\left(\frac{1}{\phi(\mu(10 Q))} \int_{Q}\left(\sum_{j \in \mathbb{N}} M f_{j, 2}(x)^{r}\right)^{\frac{p}{r}} d \mu(x)\right)^{\frac{1}{p}} \leq c\left\|\left(\sum_{j \in \mathbb{N}}\left|f_{j}\right|^{r}\right)^{\frac{1}{r}}: \mathcal{L}^{p, \phi}(\mu)\right\| .
$$

If we combine this with the estimate for the $f_{j, 1}$, the proof is now complete.

\section{Boundedness of fractional integral operators}

Now we investigate the boundedness of the fractional integral operators. In [14], we have defined a fractional integral operator $I_{\beta, \kappa}^{b}$ for general measures and investigated relationship between the one defined in [13] and $I_{\beta, \kappa}^{b}$. We define

$$
K_{\beta, \kappa}^{b}(x, y):=\sup _{x, y \in Q \in \mathcal{Q}(\mu)} \mu(\kappa Q)^{-(1-\beta)} .
$$

For a positive function $f$ we define

$$
I_{\beta, \kappa}^{b} f(x)=\int_{\mathbb{R}^{d}} K_{\beta, \kappa}^{b}(x, y) f(y) d \mu(y) .
$$


In general for a $\mu$-measurable $\mathbb{R}$-valued function, we write $f^{+}(x):=\max (f(x), 0)$ and $f^{-}(x):=f^{+}(x)-f(x)$ and set

$$
I_{\beta, \kappa}^{b} f(x)=I_{\beta, \kappa}^{b} f^{+}(x)-I_{\beta, \kappa}^{b} f^{-}(x),
$$

if $I_{\beta, \kappa}^{b} f^{+}(x)$ and $I_{\beta, \kappa}^{b} f^{-}(x)$ are both finite for $\mu$-a.e. $x \in \mathbb{R}^{d}$.

Lemma 3.1 ([13, Proposition 18], [14, Corollary 4.4]). Suppose that $\kappa>1$ and $0<\beta<1$. Then

$$
M_{\beta} f(x) \leq 2 I_{\beta, \sqrt{2}}^{b}|f|(x) \mu-a . e .
$$

for all $\mu$-measurable function $f$.

Proposition 3.2 ([13, Proposition 11, 19], [14, Corollary 4.4]). Suppose that $\mu$ is a growth measure: $\mu(B(x, r)) \leq c_{0} r^{n}$. Let $0<\alpha<n$ and $\kappa>1$. Then

$$
I_{\alpha} f(x) \leq c I_{\alpha / n, \kappa}^{b}|f|(x)
$$

for all $\mu$-measurable function $|f|$.

Theorem 3.3. Suppose that $\phi$ satisfies (7) and that

$$
\int_{r}^{\infty} \frac{\phi(t)}{t^{\frac{p}{q}}} \frac{d t}{t} \leq c \frac{\phi(r)}{r^{\frac{p}{q}}}
$$

If the parameters $p, q, \kappa, \beta$ satisfy

$$
0<\beta<1<\kappa, \quad 1<p<q<\infty, \quad \frac{1}{q}=\frac{1}{p}-\beta,
$$

then $I_{\beta, \kappa}^{b}$ is a bounded operator from $\mathcal{L}^{p, \phi}(\mu)$ to $\mathcal{L}^{q, \phi^{\frac{q}{p}}}(\mu)$

Proof. We may assume that the function $f$ is positive, since the kernel is positive. Let $Q \in \mathcal{Q}(\mu)$ be fixed. Then we decompose $f$ according to $2 K Q$. Namely, we split $f$ by $f=f_{1}+f_{2}$ with $f_{1}=\chi_{2 K Q} f$ and $f_{2}=\chi_{\mathbb{R}^{d} \backslash 2 K Q} f$, where $K$ is large enough according to the value of $\kappa$. Then we have to show

$$
\left(\frac{1}{\phi(\mu(4 K Q))^{\frac{q}{p}}} \int_{Q} I_{\beta, \kappa}^{b} f_{i}(x)^{q} d \mu(x)\right)^{\frac{1}{q}} \leq c\left\|f: \mathcal{L}^{p, \phi}(\mu)\right\|
$$

for $i=1,2$. The estimate for $i=1$ is simple. Since it is known that $I_{\beta, \kappa}^{b}$ is $L^{p}(\mu)-L^{q}(\mu)$ bounded, we have

$$
\begin{aligned}
\left(\frac{1}{\phi(\mu(4 K Q))^{\frac{q}{p}}} \int_{Q} I_{\beta, \kappa}^{b} f_{i}(x)^{q} d \mu(x)\right)^{\frac{1}{q}} & \leq c\left(\frac{1}{\phi(\mu(4 K Q))} \int_{2 K Q} f(x)^{p} d \mu(x)\right)^{\frac{1}{p}} \\
& \leq c\left\|f: \mathcal{L}^{p, \phi}(\mu)\right\| .
\end{aligned}
$$

Thus, the proof for $i=1$ is now valid. We now turn our attention to the estimate for $i=2$. We first write out $I_{\beta, \kappa}^{\mathrm{b}} f_{2}(x)$ in full:

$$
I_{\beta, \kappa}^{b} f_{2}(x)=\int_{\mathbb{R}^{d} \backslash 2 K Q}\left(\sup _{x, y \in R \in \mathcal{Q}(\mu)} \mu(\kappa R)^{-(1-\beta)}\right) f(y) d \mu(y) .
$$


A geometric observation again shows that $\sqrt{\kappa} R$ engulfs $2 Q$, the center of $Q$ in its interior, provided $K$ is large enough and $R \in \mathcal{Q}(\mu)$ intersects both $Q$ and $\mathbb{R}^{d} \backslash 2 K Q$. Therefore, $I_{\beta, \kappa}^{b} f_{2}(x)$ has a uniform upper bound:

$$
I_{\beta, \kappa}^{b} f_{2}(x) \leq c \int_{\mathbb{R}^{d} \backslash 2 K Q}\left(\sup _{\substack{R \in \mathcal{Q}(\mu) \\\{y\} \cup 2 Q \subset R}} \mu(\sqrt{\kappa} R)^{-(1-\beta)}\right) f(y) d \mu(y) .
$$

With (11) in mind, we shall partition $\mathbb{R}^{d} \backslash 2 Q$. We set, for $j \geq 1$,

$$
\mathcal{D}_{j}:=\left\{y \in \mathbb{R}^{d} \backslash 2 K Q: 2^{j-1} \mu(2 Q) \leq \inf _{\substack{R \in \mathcal{Q}(\mu) \\\{y\} \cup 2 Q \subset \operatorname{Int}(R)}} \mu(\sqrt{\kappa} R)<2^{j} \mu(2 Q)\right\},
$$

where $\operatorname{Int}(E)$ denotes the interior of a set $E$. By using Lemma 2.2, we can find a set of cubes $\left\{Q_{j}^{l}\right\}_{l \in L_{j}}$ so that

$$
2^{j-1} \mu(2 Q) \leq \mu(\sqrt{\kappa} R)<2^{j} \mu(2 Q), \quad \mathcal{D}_{j} \subset \bigcup_{l \in L_{j}} \sqrt{\kappa} Q_{j}^{l}, \quad \sharp L_{j} \leq N,
$$

where $N$ depends only on $\kappa$ and $d$. Thus, we obtain, for all $x \in Q$,

$$
\begin{aligned}
I_{\beta, \kappa}^{b} f_{2}(x) & \leq c \sum_{j \in \mathbb{N}} \sum_{l \in L_{j}} \frac{1}{\left(2^{j} \mu(2 Q)\right)^{1-\beta}} \int_{\sqrt{\kappa} Q_{j}^{l}} f(y) d \mu(y) \\
& \leq c \sum_{j \in \mathbb{N}} \sum_{l \in L_{j}} \frac{1}{\left(2^{j} \mu(2 Q)\right)^{-\beta+\frac{1}{p}}}\left(\int_{\sqrt{\kappa} Q_{j}^{l}} f(y)^{p} d \mu(y)\right)^{\frac{1}{p}} \\
& \leq c\left(\sum_{j \in \mathbb{N}} \sum_{l \in L_{j}} \frac{\phi(\mu(2 Q))^{\frac{1}{p}}}{\left(2^{j} \mu(2 Q)\right)^{\frac{1}{q}}}\right) \cdot\left\|f: \mathcal{L}^{p, \phi}(\mu)\right\| \\
& \leq c \sum_{j \in \mathbb{N}}\left(\frac{\phi(\mu(2 Q))^{\frac{1}{p}}}{\left(2^{j} \mu(2 Q)\right)^{\frac{1}{q}}}\right) \cdot\left\|f: \mathcal{L}^{p, \phi}(\mu)\right\| .
\end{aligned}
$$

If we integrate this inequality over $Q$ and arrange both sides, we obtain

$$
\left(\frac{1}{\phi(\mu(4 K Q))^{\frac{q}{p}}} \int_{Q} I_{\beta, \kappa}^{b} f_{2}(x)^{q} d \mu(x)\right)^{\frac{1}{q}} \leq c\left\|f: \mathcal{L}^{p, \phi}(\mu)\right\|
$$

by virtue of Lemma 2.1 again, which is the desired result.

Before we go further, we shall present a series of corollaries.

Corollary 3.4. Under the same assumption as Theorem 3.3, we have

$$
\left\|M_{\beta} f: \mathcal{L}^{q, \phi^{\frac{q}{p}}}(\mu)\right\| \leq c\left\|f: \mathcal{L}^{p, \phi}(\mu)\right\|,
$$


where $M_{\beta}$ is a fractional maximal operator given by

$$
M_{\beta} f(x)=\sup _{x \in Q \in \mathcal{Q}(\mu)} \frac{1}{\mu(2 Q)^{1-\beta}} \int_{Q}|f(y)| d \mu(y) .
$$

Corollary 3.5. In addition to the same assumption on $\phi$ as Theorem 3.3 , postulate $\mu$ on the growth condition (5):

$$
\mu(B(x, \ell)) \leq c_{0} \ell^{n}, \quad 0<n \leq d,
$$

where $c_{0}$ and $n$ is a fixed number. Suppose further that the parameters $p, q, \alpha$ satisfy

$$
0<\alpha<n, \quad 1<p<q<\infty, \quad \frac{1}{q}=\frac{1}{p}-\frac{\alpha}{n} .
$$

Then the fractional integral operator $I_{\alpha}$, defined by

$$
I_{\alpha} f(x)=\int_{\mathbb{R}^{d}} \frac{f(y)}{|x-y|^{n-\alpha}} d \mu(y),
$$

enjoys the following boundedness:

$$
\left\|I_{\alpha} f: \mathcal{L}^{q, \phi^{\frac{q}{p}}}\right\| \leq c\left\|f: \mathcal{L}^{p, \phi}\right\| .
$$

\section{Boundedness of singular integral operators}

In this section we consider singular integral operator. Throughout this section we assume that $\mu$ is a growth measure satisfying (5). Recall that the singular integral operator $T$ is a bounded linear operator from $L^{2}(\mu)$ to $L^{2}(\mu)$ that satisfies the following: There exists a function $K$ that satisfies three properties listed below.

(1) There exists $c>0$ such that

$$
|K(x, y)| \leq \frac{c}{|x-y|^{n}}
$$

(2) There exist $\varepsilon>0$ and $c>0$ such that

$$
|K(x, y)-K(z, y)|+|K(y, x)-K(y, z)| \leq c \frac{|x-z|^{\varepsilon}}{|x-y|^{n+\varepsilon}},
$$

whenever $|x-y| \geq 2|x-z|$.

(3) If $f$ is a compactly supported bounded $\mu$-measurable function, then

$$
T f(x)=\int_{\mathbb{R}^{d}} K(x, y) f(y) d \mu(y) \quad \text { for all } \quad x \notin \operatorname{supp}(f) .
$$

Theorem 4.1. Suppose that $1<p<\infty$ and $T$ is a singular integral operator as above. Assume $\phi$ is a function satisfying (7), the doubling condition

$$
\sup _{\substack{r, s>0 \\ r \leq s \leq 2 r}} \frac{\phi(r)}{\phi(s)}<\infty
$$


and

$$
\int_{r}^{\infty} \frac{\phi(t)}{t} \frac{d t}{t} \leq c \frac{\phi(r)}{r}
$$

Then we have

$$
\left\|T f: \mathcal{L}^{p, \phi}(\mu)\right\| \leq c\left\|f: \mathcal{L}^{p, \phi}(\mu)\right\| .
$$

Proof. Fix a cube $Q \in \mathcal{Q}(\mu)$ as before. Then we have to estimate

$$
\left(\frac{1}{\phi(\mu(4 Q))} \int_{Q}|T f(x)|^{p} d \mu(x)\right)^{\frac{1}{p}} \leq c\left\|f: \mathcal{L}^{p, \phi}(\mu)\right\| .
$$

Decompose $f$ according to $2 Q$, that is, we now split $f$ by $f=f_{1}+f_{2}$ with $f_{1}=\chi_{2 Q} \cdot f$ and $f_{2}=\chi_{\mathbb{R}^{d} \backslash 2 Q} \cdot f$. Then along this decomposition the estimate (17) can be separated into

$$
\begin{aligned}
& \left(\frac{1}{\phi(\mu(4 Q))} \int_{Q}\left|T f_{1}(x)\right|^{p} d \mu(x)\right)^{\frac{1}{p}} \leq c\left\|f: \mathcal{L}^{p, \phi}(\mu)\right\| \\
& \left(\frac{1}{\phi(\mu(4 Q))} \int_{Q}\left|T f_{2}(x)\right|^{p} d \mu(x)\right)^{\frac{1}{p}} \leq c\left\|f: \mathcal{L}^{p, \phi}(\mu)\right\| .
\end{aligned}
$$

The estimate for $f_{1}$ is easy because $T$ is $L^{p}(\mu)$-bounded. We now turn our attention to the estimate for $f_{2}$. Denoting by $c_{Q}$ the center of $Q$, we obtain a pointwise estimate with the aid of (12) as before:

$$
\left|T f_{2}(x)\right| \leq c \int_{\mathbb{R}^{d} \backslash 2 Q} \frac{|f(y)|}{\left|y-c_{Q}\right|^{n}} d \mu(y) .
$$

We insert an equality $n \int_{0}^{\infty} \frac{\chi_{B(x, \ell)}(y)}{\ell^{n+1}} d \ell=\frac{1}{|x-y|^{n}}$ to (18) and change the order of integration. Then we obtain

$$
\left|T f_{2}(x)\right| \leq c \int_{2 \ell(Q)}^{\infty} \frac{1}{\ell^{n+1}}\left(\int_{B\left(c_{Q}, \ell\right)}|f(y)| d \mu(y)\right) d \ell .
$$

By using the Hölder inequality and the assumption we have

$$
\begin{aligned}
\left|T f_{2}(x)\right| & \leq c\left(\int_{2 \ell(Q)}^{\infty} \frac{\phi\left(c \ell^{n}\right)^{\frac{1}{p}}}{\ell^{\frac{n}{p}+1}} d \ell\right) \cdot\left\|f: \mathcal{L}^{p, \phi}(\mu)\right\| \\
& \leq c\left(\frac{\phi\left(c \ell^{n}\right)}{\ell^{n}}\right)^{\frac{1}{p}} \cdot\left\|f: \mathcal{L}^{p, \phi}(\mu)\right\| .
\end{aligned}
$$

Since $\phi$ is a doubling function satisfying (15) and we are assuming the almostdecreasing condition (7) and the doubling condition (15), we have

$$
\left|T f_{2}(x)\right| \leq c\left(\frac{\phi\left(\mu\left(B\left(c_{Q}, 2 d \ell\right)\right)\right.}{\mu\left(B\left(c_{Q}, 2 d \ell\right)\right)}\right)^{\frac{1}{p}} \cdot\left\|f: \mathcal{L}^{p, \phi}(\mu)\right\| .
$$

Integrating this over $Q$, we obtain the desired estimate. 
Finally we remark that we can make the vector-valued extension of the results obtained in this paper other than the maximal operator $M$. The vector valued extension of $M$ is somehow non-trivial. For details we refer to $[13,15]$.

\section{References}

[1] D. Adams, A note on Riesz potentials, Duke Math. J. 42 (1975), 765-778.

[2] D. Edmunds, V. Kokilashvili and A. Meskhi, Bounded and compact integral operators. Kluwer Academic Publishers, Dordrecht, Boston London, 2002.

[3] J. García-Cuerva and E. Gatto, Boundedness properties of fractional integral operators associated to non-doubling measures, Studia Math. 162 (2004), no. 3, 245-261.

[4] J. García-Cuerva and J.M. Martell, Weighted inequalities and vector-valued Calderón-Zygmund operators on nonhomogeneous spaces, Publ. Mat. 44 (2000), no. $2,613-640$.

[5] T. Mizuhara, Commutators of Singular Integral Operators on Morrey spaces with General Growth Functions, Suurikaiseki Koukyuroku. 1102 (1999), 49-63.

[6] T. Mizuhara, Commmutator of singular integral operators on Morrey spaces with some growth functions, Proceedings of the Second ISAAC Congress, 1, 65-73.

[7] C. Morrey, On solutions of quasi-linear elliptic partial differential equations, Trans. Amer. Math. Soc. 43 (1938), 126-166.

[8] E. Nakai, Generalized fractional integrals on Orlicz-Morrey spaces, Proceedings of the International Symposium on Banach and Function spaces, Yokohama Publishers, (2004), 323-333.

[9] E. Nakai, Hardy-Littlewood maximal operator, singular integral operators and the Riesz potentials on generalized Morrey spaces, Math. Nachr. 166 (1994), 95-103.

[10] F. Nazarov, S. Treil and A. Volberg, Cauchy integral and Calderón-Zygmund operators on nonhomogeneous spaces, Internat. Math. Res. Notices (1997), no. 15, $703-726$

[11] F. Nazarov, S. Treil and A. Volberg, Weak type estimates and Cotlar inequalities for Calderön-Zygmund operators on nonhomogeneous spaces, Internat. Math. Res. Notices (1998), no. 9, 463-487.

[12] Y. Sawano, Sharp estimates of the modified Hardy-Littlewood maximal operator on the nonhomogeneous space via covering lemmas, Hokkaido Math. J. 34 (2005), $435-458$

[13] Y. Sawano, $l^{q}$-valued extension of the fractional maximal operators for non-doubling measures via potential operators, International Journal of Pure and Applied mathematics, 26 (2006), no. 4, 505-523.

[14] Y. Sawano, T. Sobukawa and H. Tanaka, Limiting case of the boundedness of fractional integral operators on non-homogeneous space, Journal of Inequalities and Applications, vol. 2006, Article ID 92470, 16 pages, 2006.

[15] Y. Sawano and H. Tanaka, Morrey spaces for non-doubling measures, Acta Math. Sinica 21 (2005), no. 6, 1535-1544.

[16] S. Shirai, Notes on commutators of fractional integral operators on generalized Morrey spaces, Scientiae Mathematicae Japonicae, e-2006, 113-118. 
[17] X. Tolsa, BMO, $H^{1}$, and Calderón-Zygmund operators for non doubling measures, Math. Ann. 319 (2001), 89-149.

[18] X. Tolsa, The space $H^{1}$ for nondoubling measures in terms of a grand maximal operator, Trans. Amer. Math. Soc. 355 (2003), no. 1, 315-348 (electronic).

Yoshihiro Sawano

Department of Mathematics

Gakushuin University

1-5-1 Mejiro, Toshima-ku

Tokyo 171-8588

Japan

e-mail: yosihiro@math.gakushuin.ac.jp

Received: 8 November 2006.

Accepted: 30 May 2007. 